\title{
Tailing effect of PD-1 antibody results in the eradication of unresectable multiple primary lung cancer presenting as ground-glass opacities: a case report
}

\author{
Shibo Wu ${ }^{1 \#}$, Dan $\mathrm{Li}^{2 \#}$, Jinping Chen ${ }^{3}$, Weizhuang Chen ${ }^{1}$, Feng $\operatorname{Ren}^{4}$ \\ ${ }^{1}$ Department of Respiratory Medicine, Lihuili Hospital, Ningbo Medical Center, Ningbo, China; ${ }^{2}$ Department of Cardiology, The Second Hospital \\ of Yinzhou, Ningbo, China; ${ }^{3}$ Department of Diagnosis, Ningbo Diagnostic Pathology Center, Ningbo, China; ${ }^{4}$ Department of Medical Imaging, \\ Lihuili Hospital, Ningbo Medical Center, Ningbo, China \\ \#These authors contributed equally to this work. \\ Correspondence to: Feng Ren. Department of Medical Imaging, Lihuili Hospital, Ningbo Medical Center, No. 57, Xing Ning Road, Ningbo 315041, \\ China. Email: rxyrf148@sina.com.
}

\begin{abstract}
There is currently no standard treatment for multiple primary lung cancer (MPLC). We report a case of synchronous MPLC presenting as one ground-glass opacity (GGO) with predominant consolidation accompanied by at least parietal pleura involvement, and another with >30 GGOs distributed across bilateral lungs, which was ineligible for complete resection. CT-guided percutaneous biopsy of the nearly pure-solid mass showed invasive lung adenocarcinoma mainly composed of acinar type. Capture-based, ultra-deep targeted sequencing (Burning Rock, Guangzhou, China) was performed on the tumor tissue biopsy. The result revealed no druggable mutations according to the guideline and a high TMB of 34.1 Mb. Immunohistochemical staining (22C3; Dako, Denmark) was positive for PD-L1 expression with a tumor expression level of $30 \%$. Based on the clinical information and patient's decision, he received 3 cycles of pemetrexed plus pembrolizumab and was subsequently forced to withdraw due to acquired immune-related pneumonitis. After discontinuation of corticosteroids, he was subjected to wedge resection for the nearly pure-solid lesion, and then refused further treatment for the other tumors. After a follow-up of 12 months from termination of immunotherapy, almost all GGOs achieved radiographically complete remission, attributed to the tailing effect of the programmed cell death protein 1 (PD-1) antibody of pembrolizumab. Through the case study we found that unresectable synchronous MPLC presenting as GGOs may respond well to immunotherapy.
\end{abstract}

Keywords: Multiple primary lung cancer (MPLC); pembrolizumab; tailing effect; i; ground-glass opacities (GGOs); case report

Submitted Sep 27, 2020. Accepted for publication Jan 19, 2021.

doi: 10.21037/apm-20-2132

View this article at: http://dx.doi.org/10.21037/apm-20-2132

\section{Introduction}

Multiple primary lung cancer (MPLC) was first reported in 1924 (1), and its incidence had increased in recent years, most likely due to advances in high-resolution detection technology (2). Curative treatments, such as surgical resection or stereotactic ablative radiotherapy, have their limitation $(3,4)$. He et al. launched a phase II, prospective design study on July 24, 2019, which was the first worldwide attempt to test the programmed cell death protein 1 (PD-1)

\footnotetext{
$\wedge$ ORCID: 0000-0003-2449-2903.
} 
antibody, sintilimab, on unresectable multiple "stage 0" lung cancer with ground-glass opacities (GGOs) (5). Herein, we describe a case of MPLC characterized as widespread GGOs in different sizes and a consolidation ratio in bilateral lungs. The patient was administrated 3 cycles of pemetrexed combined with pembrolizumab until the onset of immune-related pneumonitis. Two weeks after the treatment of immune-related pneumonitis, the GGO with predominant consolidation was removed and the patient did not achieve major pathological response (MPR) at that time. Computed tomography (CT) scan 12 months after the termination of immunotherapy and chemotherapy showed that almost all of the remaining GGOs achieved complete response. The morphology, tumor mutation burden (TMB), PD-L1 expression, and tumor immune microenvironment (TIME) of the samples before and after treatment were compared and analyzed in detail. In this case, we demonstrated the outstanding efficacy of pembrolizumab on MPLC presenting as GGOs, and explored the mechanism underlying the tailing effect of pembrolizumab.

We present the following article in accordance with the CARE reporting checklist (available at http://dx.doi. org/10.21037/apm-20-2132).

\section{Case presentation}

A 55-year-old male, who was a heavy smoker, presented with a complaint of left chest pain for months; he hospitalized in April 2019. As well as emphysema, chest contrast-enhanced CT revealed widespread GGOs in the bilateral lungs with a predominately solid mass measuring $2 \times 2 \mathrm{~cm}$ in diameter accompanied by at least parietal pleura involvement in the left upper lung (Figure 1A). In addition, there was no other neoplasm-associated metastasis observed on cranial contrast-enhanced magnetic resonance imaging. Carcinoembryonic antigen (CEA) and squamous cell carcinoma antigen (SCC) were $11.3 \mu \mathrm{g} / \mathrm{L}$ (normal 0-5 $\mu \mathrm{g} / \mathrm{L}$ ) and $2.79 \mu \mathrm{g} / \mathrm{L}$ (normal 0-2.5 $\mu \mathrm{g} / \mathrm{L}$ ), respectively. CT-guided percutaneous biopsy of the nearly pure-solid mass showed invasive lung adenocarcinoma mainly composed of acinar type. Our multidisciplinary team agreed on the diagnosis of MPLC; however, complete resection of all lesions was not possible.

The patient provided informed consent, and capturebased, ultra-deep targeted sequencing (Burning Rock, Guangzhou, China) was performed on the tumor tissue biopsy using a panel consisting of 520 cancer-related genes, spanning 1.6 Mb of human genome. The result revealed no druggable mutations according to the guideline and a high TMB of 34.1 Mb. Immunohistochemical staining (22C3; Dako, Denmark) was positive for PD-L1 expression with a tumor expression level of $30 \%$. Based on the clinical information and patient's decision, mono-chemotherapy of pemetrexed combined with pembrolizumab was administered as first-line therapy on May 25, 2019. After 3 cycles of the therapy, the patient developed pyrexia (with his highest temperature being $39.5^{\circ} \mathrm{C}$ ), dry cough, dyspnea, and fatigue on July 19, 2019. Multiple new-onset diffuse patchy shadows and ground-glass appearances were observed to be distributed across the bilateral lungs on CT imaging, despite a reduction in the size and density of MPLC (Figure $1 \mathrm{~B}$ ). Normal white blood count and a significant increase in erythrocyte sedimentation rate $(82 \mathrm{~mm} / \mathrm{h}$ vs. normal $<15 \mathrm{~mm} / \mathrm{h})$ and C-reactive protein level (69.2 mg/L $v s$. normal $<5 \mathrm{mg} / \mathrm{L}$ ) were detected. The patient's clinical symptoms, imaging, blood test results and no response to the antibiotic therapy in 5 days were taken into consideration, and was diagnosed with grade 3 immune-related pneumonitis. In addition to immunotherapy cessation, he was administrated $0.5 \mathrm{mg} / \mathrm{kg} /$ day prednisone and soon achieved significant alleviation in symptoms, and improvements were observed in his imaging results. The prednisone dose was gradually tapered within 4 weeks. The multidisciplinary team recommended palliative resection of the predominate solid mass. With no recurrence of immune-related pneumonitis observed after discontinuation of corticosteroids, he was subjected to wedge resection of the left upper lung.

Postoperative morphology indicated invasive adenocarcinoma of mainly papillary type, followed by acinar type; MPR was not achieved. The patient received no further treatment for the residual tumors following surgery. Chest CT and a blood test for serum CEA and SCC levels were performed at 3-month intervals. Radiographic findings indicated that almost all of the remaining GGOs gradually achieved complete remission (Figure 1C), while serum CEA and SCC were restored to normal range 12 months after discontinuation of immunotherapy.

Morphology, TMB, PD-L1 expression, and TIME analyses of the samples before and after treatment were performed using the same detection technique. TMB of the pretreatment tumor sample was 34.1 Mb compared with 26.2 $\mathrm{Mb}$ of the postoperative tumor sample. Mutant allele frequencies and variants not detected in the corresponding sample are shown for each specimen in Table 1. The TIME of samples pre- and post-treatment had similar 

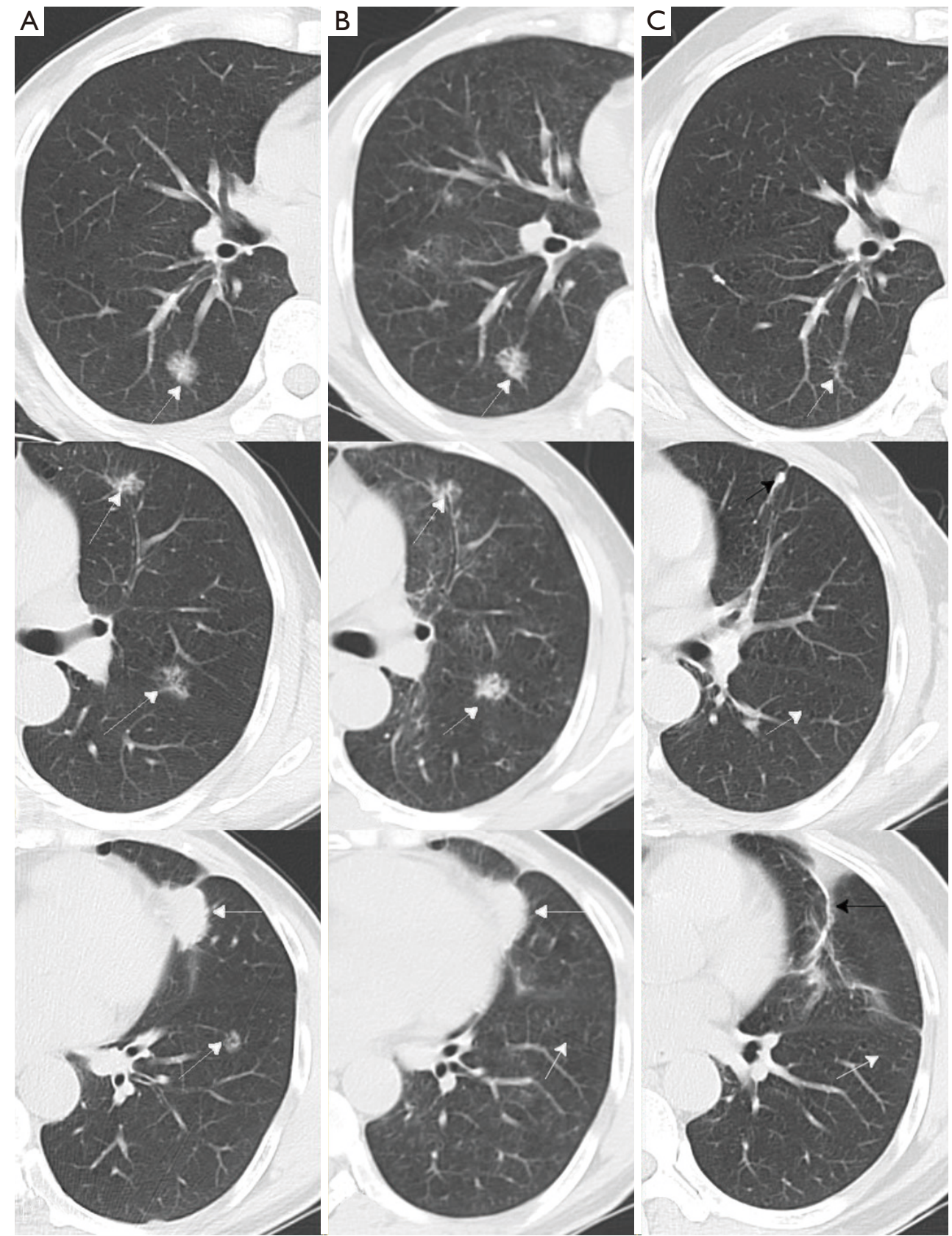

Figure 1 Typical CT imaging features before and after treatment. (A) Five typical ground-glass opacities (GGOs) (white arrow) with varying sizes and consolidation ratio in the bilateral lungs were observed on computed tomography (CT) pretreatment. (B) After 3 cycles of chemotherapy plus immunotherapy, a reduction in the size and density of typical GGOs (white arrow) was observed radiographically. In addition, there were new-onset diffuse patchy shadows and ground-glass appearances that were observed to be distributed across the bilateral lungs on CT. (C) CT revealed normal postoperative change in the left lingula lobe (black arrow), and eradication of the other typical GGOs (white arrow) contrasted with previous imaging 12 months following immunotherapy cessation. 
Table 1 Gene mutations and calculated tumor mutation burden of the biopsy specimen sample and surgery specimen

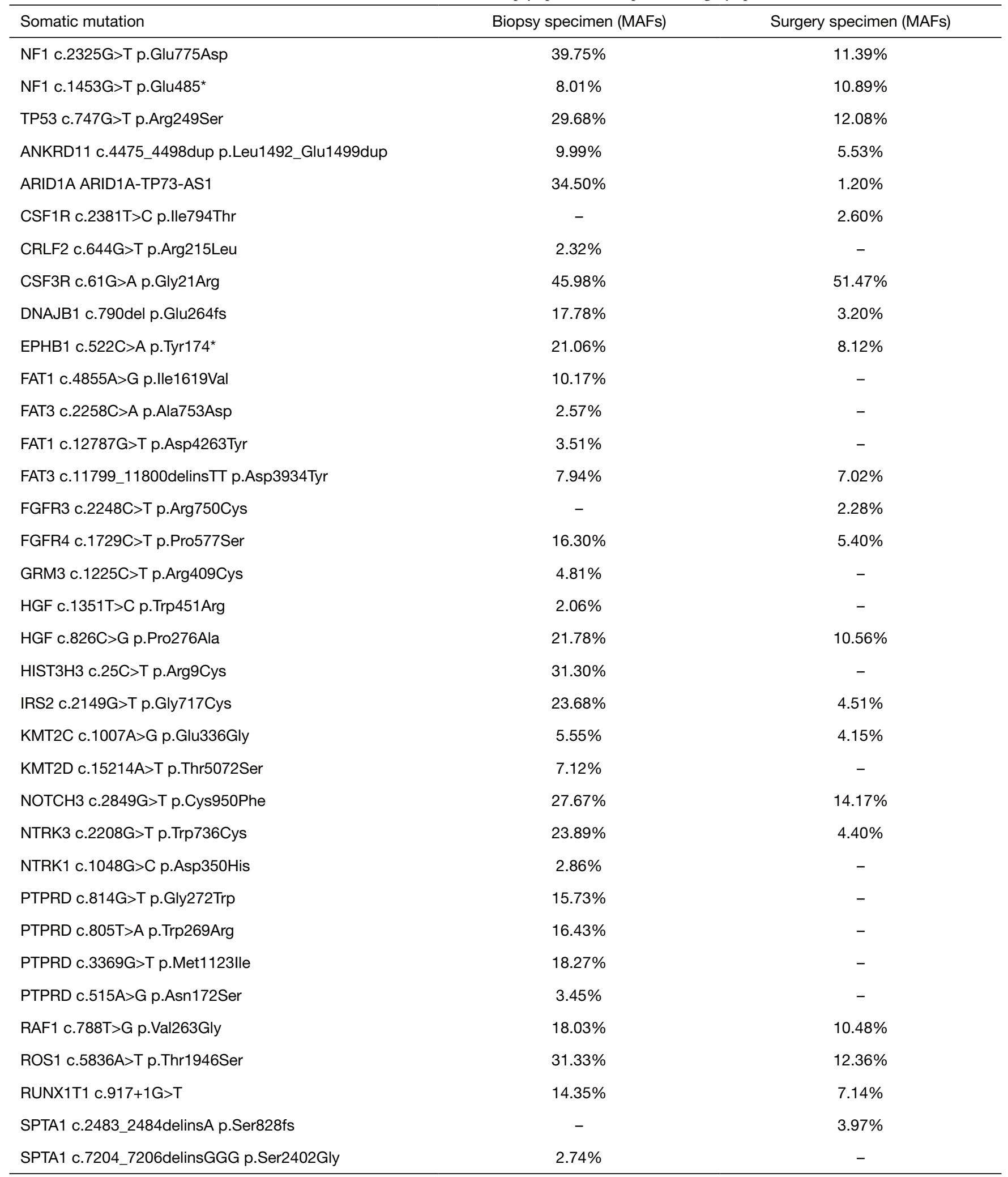

Table 1 (continued) 
Table 1 (continued)

\begin{tabular}{lcc}
\hline Somatic mutation & Biopsy specimen (MAFs) & Surgery specimen (MAFs) \\
\hline TBX3 c.1448C>A p.Thr483Lys & $27.15 \%$ & $7.60 \%$ \\
TBX3 c.137T>C p.Leu46Pro & - & $2.40 \%$ \\
U2AF1 c.101C>T p.Ser34Phe & $17.20 \%$ & $9.55 \%$ \\
XRCC2 c.21G>T p.Arg7Ser & - & $3.05 \%$ \\
TMB (mutations/Mb) & 34.1 & 26.2 \\
\hline
\end{tabular}

Mutant allele frequencies (MAFs) are shown for each specimen. Slash (-) indicates that the variant was not detected in the corresponding sample.

characteristics, including PD-L1 tumor expression of 50\% and $30 \%$, respectively, and tumor infiltrating lymphocyte (TIL) cell infiltration in the tumor parenchyma and stroma in varying degrees (Figure 2). The method of TIME as follows: To identify the CD8+, CD3+, PD-1, and PD-L1-expressing cells from the TIME, multiplex immunofluorescence staining using PANO 4-plex IHC kit (Panovue, Beijing, China) was performed according to manufacturer's protocol. Nuclei were stained with 4'-6'-diamidino-2-phenylindole (DAPI, Sigma-Aldrich, St. Louis, Missouri, USA). To obtain multispectral images, the stained slides were scanned using the Mantra System (PerkinElmer, Waltham, Massachusetts, USA). Images of unstained and single-stained sections were used to extract the spectrum of autofluorescence of tissues and each fluorescein, respectively. Further image processing was performed using inform advanced image analysis software (Perkin Elmer, Waltham, Massachusetts, USA). All procedures performed in studies involving human participants were in accordance with the ethical standards of the institutional and/or national research committee(s) and with the Helsinki Declaration (as revised in 2013). Written informed consent was obtained from the patient.

\section{Discussion}

We presented a case with unresectable synchronous MPLC, which presented as multiple GGOs in iconography corresponding to in situ, microinvasive, or invasive adenocarcinoma in pathology in terms of the proportion of solid components. The majority of the GGOs achieved complete response due to the tailing effect of pembrolizumab during follow-up. TMB and PD-L1 expressions were independent variables, and a composite of TMB plus PD-L1 further enriched for benefit to immune checkpoint inhibitors (6). The TIME can be divided into 4 types, based on the TIL combined with PD-L1 expression. Type II (PD-L1+ and TIL+) was proposed to be the most responsive to the PD-1 antibody (7). In our case, GGO with predominant consolidation with high TMB, positive PD-L1 expression, and type II TIME, according to the aforementioned classification, indicated that the patient might response to anti-PD-1 treatment. Based on the complete remission of the remaining GGOs 12 months after pneumonitis, we hypothesized that TMB or the immune regulation of these GGOs with a varying consolidation ratio might mimic that of the GGO with a predominantly solid mass. Although postoperative specimens did not achieve MPR 6 weeks after immunotherapy, we observed a significant reduction in the lesions radiographically prior to surgery and considered them suitable for immunotherapy.

Nelson et al. observed differential immune cell infiltration and PD-L1 expression in adenocarcinoma presenting as GGOs compared with solid lesions in a CT scan (8). Lung adenocarcinoma with GGO was correlated with a lower incidence of PD-L1 expression than pure-solid tumors (9). In our case, following a complete reduction in the size and density of almost all the remaining GGOs 12 months after pneumonitis, we presumed that all the GGOs had a similar TMB or TIME. It is still unclear whether adenocarcinoma presenting as GGOs has a distinct immune regulation that demonstrates varying susceptibility to immunotherapy. Available clinical and fundamental research about the pathogenic and immunoregulatory mechanism of the disease is necessary. Hence we can better understand the advantages and disadvantages including the adverse reactions of immunotherapy on MPLC presenting as GGOs.

One of the limitations of the present study was that we failed to detect TMB and immune regulation of the remaining GGOs. Due to outstanding efficacy of the 


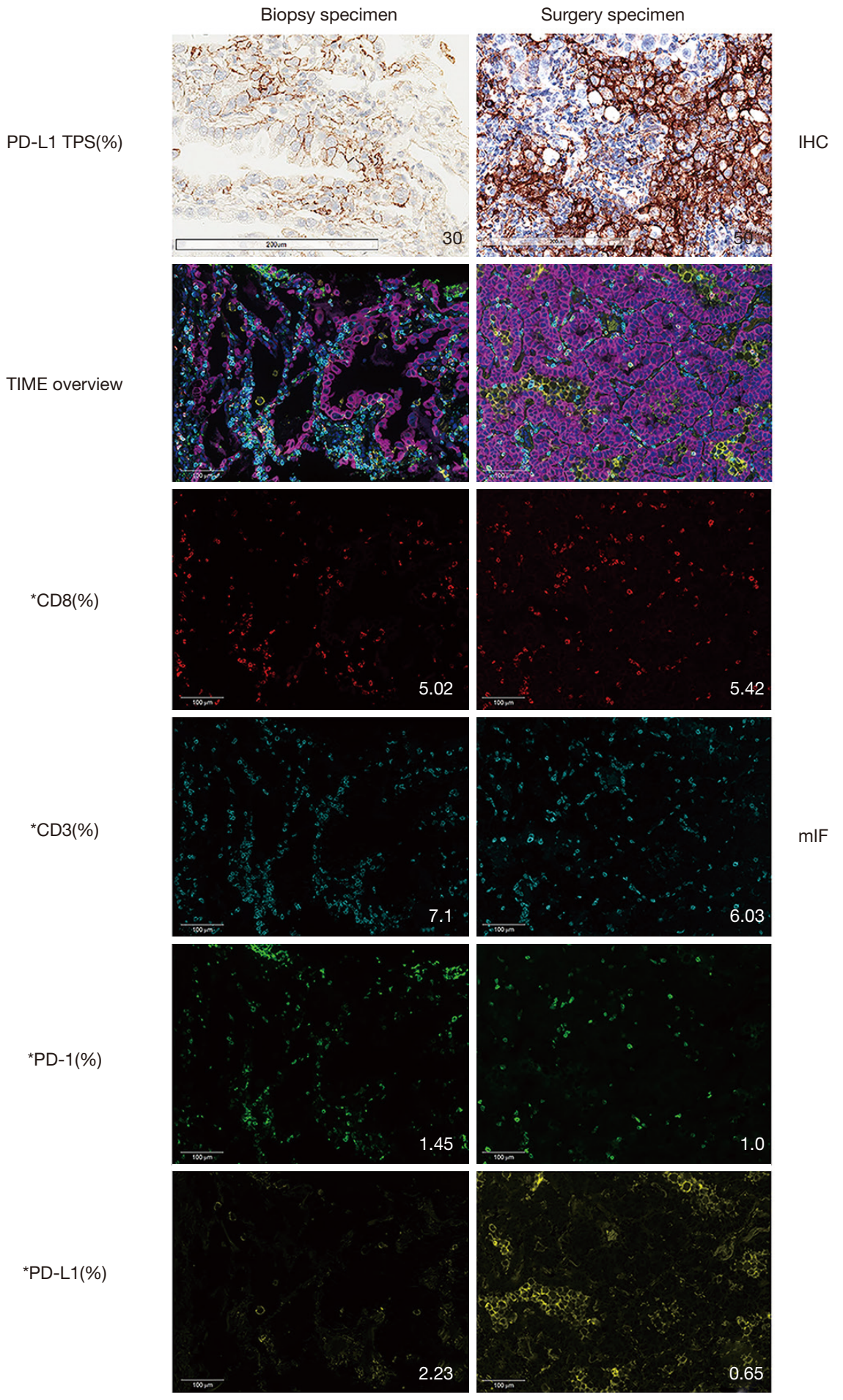

Figure 2 Tumor immune microenvironments (TIME) of the biopsy specimen sample and surgical specimen. Red, CD8; cyan, CD3; green, PD-L1; yellow, PD-L1; purple, panCK; blue, DAPI. *, assessments were focused on the tumor parenchyma regions. IHC, immunohistochemistry; mIF, multiplex immunofluorescence; PD-1, programmed cell death protein 1. 
remaining GGOs, we assumed that these GGOs had similar $\mathrm{TMB}$ and immune regulation to the resected one.

In conclusion, unresectable synchronous MPLC presenting as GGOs may respond well to immunotherapy. Further research on TMB and immune regulation detection at those respective lesions is still needed to investigate immune regulatory markers of GGOs to determine whether MPLC with GGOs demonstrates varying susceptibility to immune checkpoint inhibitors.

\section{Acknowledgments}

We are grateful to Burning Rock, Guangzhou, China for their assistance with the molecular profiling.

\section{Footnote}

Reporting Checklist: The authors have completed the CARE reporting checklist. Available at http://dx.doi.org/10.21037/ apm-20-2132.

Conflicts of Interest: All authors have completed the ICMJE uniform disclosure form (available at http://dx.doi. org/10.21037/apm-20-2132). The authors have no conflicts of interest to declare.

Ethical Statement: The authors are accountable for all aspects of the work in ensuring that questions related to the accuracy or integrity of any part of the work are appropriately investigated and resolved. All procedures performed in studies involving human participants were in accordance with the ethical standards of the institutional and/or national research committee(s) and with the Helsinki Declaration (as revised in 2013). Written informed consent was obtained from the patient.

Open Access Statement: This is an Open Access article distributed in accordance with the Creative Commons Attribution-NonCommercial-NoDerivs 4.0 International License (CC BY-NC-ND 4.0), which permits the noncommercial replication and distribution of the article with the strict proviso that no changes or edits are made and the original work is properly cited (including links to both the formal publication through the relevant DOI and the license). See: https://creativecommons.org/licenses/by-nc-nd/4.0/.

\section{References}

1. Beyreuther H. Multiplicität von Carcinomen bei einem Fall von sog. "Schneeberger" Lungenkrebs mit Tuberkulose. Archiv für Pathologische Anatomie und Physiologie und für Klinische Medicin 1924;250:230-43.

2. Trousse D, Barlesi F, Loundou A, et al. Synchronous multiple primary lung cancer: an increasing clinical occurrence requiring multidisciplinary management. J Thorac Cardiovasc Surg 2007;133:1193-200.

3. Bhaskarla A, Tang PC, Mashtare T, et al. Analysis of second primary lung cancers in the SEER database. J Surg Res 2010;162:1-6.

4. Chang JY, Liu YH, Zhu Z, et al. Stereotactic ablative radiotherapy: a potentially curable approach to early stage multiple primary lung cancer. Cancer 2013;119:3402-10.

5. Editorial Office. The first attempt worldwide to test PD-1 antibody on multiple "Stage 0 " lung cancer. Transl Lung Cancer Res 2019;8:556-7.

6. Rizvi H, Sanchez-Vega F, La K, et al. Molecular Determinants of Response to Anti-Programmed Cell Death (PD)-1 and Anti-Programmed Death-Ligand 1 (PD-L1) Blockade in Patients With Non-Small-Cell Lung Cancer Profiled With Targeted Next-Generation Sequencing. J Clin Oncol 2018;36:633-41.

7. Sanmamed MF, Chen L. A Paradigm Shift in Cancer Immunotherapy: From Enhancement to Normalization. Cell 2018;175:313-26.

8. Nelson DB, Mitchell KG, Wang J, et al. Immune regulatory markers of lepidic-pattern adenocarcinomas presenting as ground glass opacities. J Thorac Dis 2020;12:329-37.

9. Suda K, Shimoji M, Shimizu S, et al. Comparison of PDL1 Expression Status between Pure-Solid Versus PartSolid Lung Adenocarcinomas. Biomolecules. 2019;9:456.
Cite this article as: $\mathrm{Wu} \mathrm{S,} \mathrm{Li} \mathrm{D,} \mathrm{Chen} \mathrm{J,} \mathrm{Chen} \mathrm{W,} \mathrm{Ren} \mathrm{F.}$ Tailing effect of PD-1 antibody results in the eradication of unresectable multiple primary lung cancer presenting as ground-glass opacities: a case report. Ann Palliat Med 2021;10(1):778-784. doi: 10.21037/apm-20-2132 